\title{
Dynamic indentation on layered polypropylene foams
}

\author{
L. Maheo and P. Viot ${ }^{\mathrm{a}}$ \\ Arts et Metiers ParisTech, I2M-DuMAS UMR 5295, 33400 Talence, France
}

\begin{abstract}
Foams, and particularly the polypropylene foam, are more and more often used in the area of injury protection and passive safety for its energy absorption capacity. This multi-scale material is constituted of mesoscopic beads with a large variability of the material properties. First, to study the effects of these mesoscopic heterogeneities on both the macroscopic and the local behaviors, numerical simulations on virtual volumes of foam under dynamic loading have been performed. The influence of the organized system of heterogeneities has also been studied in the cases of a random distribution and a multilayered volume. Experimental dynamic compressive tests have been performed on multi-layered volumes of foam and compared with the results of the Finite Element Method. Second, indentation experiments have also been performed using a cylindrical shape indenter.
\end{abstract}

\section{Introduction}

Foams are more and more often used as a cushioning structure in order to simultaneously protect against both high and low energy impacts [1]. Following a first elastic stage, a great amount of energy can be dissipated by foams during the stress plateau [2].

Recently, energy-absorption capacity of foams has been enhanced by using layered [3] or functionally graded [4] foams. Authors of previous studies showed the interest of this kind of multi-layered foams in the area of injury protection. In order to match some loading conditions which occurs during some impacts, indentation into polymeric foams has also been studied by $[1,5,6]$.

Polypropylene (PP) foam of this study is constituted of large mesoscopic beads and microscopic cells [7]. This kind of cellular material shows a multi-scale structure where the millimetric beads (about 2-5 mm) are themselves constituted of microscopic closed cells. Three scales can therefore be defined: the macroscopic scale for the foam sample, the mesoscopic one for the beads and the microscopic one for the cells. These microscopic and mescopic structures have an influence on the macroscopic behavior.

In this present study, authors proposed to study the behavior of homogeneous and multi-layered PP foams at the macroscopic and the mesoscopic scales under dynamic loadings. The key point of this research work is to determine the influence of the scale of variability - mesoscopic (variability in the beads) or macroscopic (variability in the layers of the foam) - on the macroscopic behavior. Experimental investigations had been led by Bouix et al. [8] to characterize the behavior of the foam subjected to uniaxial compressive tests at different strain rates and for different values of density.

The first numerical part of this work consisted in studying some virtual samples of foam under dynamic compression (with the Finite Element code LS-DYNA) in order to determine numerically if the presence of heterogeneities, i.e. the mesoscopic beads, can modify the mesoscopic and the macroscopic behaviors. The mechanisms of the

a e-mail: philippe.viot@ensam.eu deformation localization in the beads of the structure have therefore been modeled by defining some zones where the material properties are adjusted in function of the local density. Their influence on the strain localization and the dynamic behavior has therefore been studied to understand the mechanical effects between the two scales.

The second part of this study deals with the capacity of multi-layered foams to dissipate energy under dynamic compressive tests. Three kinds of these foams have been tested experimentally with an original Flywheel device and numerically with the LS-Dyna software. The experimental and numerical results are presented and discussed.

Experimental indentation tests have therefore been performed to evaluate the behavior of these kinds of foams in the case of a structure test. The results are presented in a third part and show good agreement with the previous study.

\section{Numerical modelling of the material}

The foam used in this study is an expanded polypropylene (EPP) material which is constituted of millimetric porous beads (Fig. 1). A previous experimental study conducted by Bouix et al. [8] had provided us a large dataset of stress-strain curves for different values of density (34 to $150 \mathrm{~kg} \cdot \mathrm{m}^{-3}$ ) and for a large range of strain rates. A Zwick electromechanical testing machine was used for quasistatic compression tests where the strain rate was about to $10^{-1} \mathrm{~s}^{-1}$. Dynamic compressive tests were performed with viscoelastic Split Hopkinson Pressure Bars to reach strain rates of $10^{3} \mathrm{~s}^{-1}$. The experiments were completed using a Flywheel device for the intermediate strain rates [8,9].

Thanks to these many experimental data, authors decided to study the dynamic behavior of this multiscale material by using numerical virtual volumes of foam (Fig. 1) instead of more experiments. The virtual volume is constituted of heterogeneities localized randomly in an homogeneous cube of $30 \times 30 \times 30 \mathrm{~mm}^{-3}$. The influence of the heterogenities inside the virtual volume can therefore be easily studied at the macroscopic and mesoscopic scales. The software program which has been developped for this 

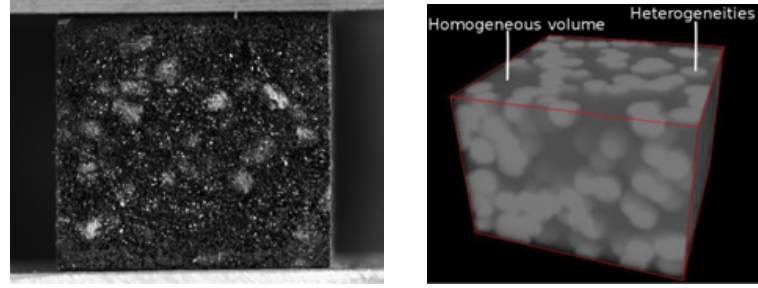

Fig. 1. Sample of a $90 \mathrm{~kg} \cdot \mathrm{m}^{-3}$ polypropylene foam under compression. Virtual volume of foam consituted of an homogeneous volume and density heterogeneities.

Table 1. Characteristics of the studied virtual samples.

\begin{tabular}{|c|c|c|c|c|}
\hline & $\begin{array}{c}\text { Size } \\
\text { Number }\end{array}$ & {$[\mathrm{mm}]$} & $\begin{array}{l}\text { Volume ratio of } \\
\text { Localization }\end{array}$ & heterogeneities [\%] \\
\hline \#1 & 337 & 3.6 & randomly & 16 \\
\hline$\# 2$ & 695 & 7.2 & randomly & 29 \\
\hline \#3 & 354 & 7.2 & randomly & 71 \\
\hline \#4 & 125 & 3.6 & middle & 6 (17 locally) \\
\hline \#5 & 1747 & 3.6 & middle & 48 (100 locally) \\
\hline
\end{tabular}

study creates virtual volumes by defining some stacks of RGB-images. The grey level of the images is only for display purposes representing the different densities. The influence of the size and the location of the heterogeneities have been studied. To do so, several kinds of virtual samples have been, three per kind of sample, to check repeatability and the representative samples are listed in the Table 1.

A difference in density of $50 \mathrm{~kg} \cdot \mathrm{m}^{-3}$ has been arbitrarly applied between the heterogeneities and the rest of the volume with heavier heterogeneities in order to use different macroscopic behaviors [8]. In order to compare virtual volumes with each other, their mean density has been globally adjusted to reach a density value of $100 \mathrm{~kg} \cdot \mathrm{m}^{-3}$. In the Table 1 , the ratio of the heterogeneities volume over the total volume is also given. This variable is interesting to be considered in order to differentiate the samples with each other.

The virtual samples of foam are then discretized into finite elements. The software program developped for this study calculates the mean of RGB value of each voxel included in each finite element. This operation means to give 256 different material properties to each finite element. The grey level of the picture gives therefore a display of the density.

The numerical simulations have therefore been performed on the LS-Dyna software [10]. The behavior law used for this study is the *MAT_FU_CHANG_FOAM[11] which describes the behavior of non-crushable foams. As well as using basic parameters (i.e., the density and the Young modulus), this law needs data such as the strainstress curves at different strain rates in order to model rate effects. The curves obtained by Bouix et al. [8] have been used for the current study.

First, the uni-axial compression of virtual samples of foam has been studied. An axial velocity of $3.9 \mathrm{~m} \cdot \mathrm{s}^{-1}$ with a maximum displacement of $21 \mathrm{~mm}$ was applied on the

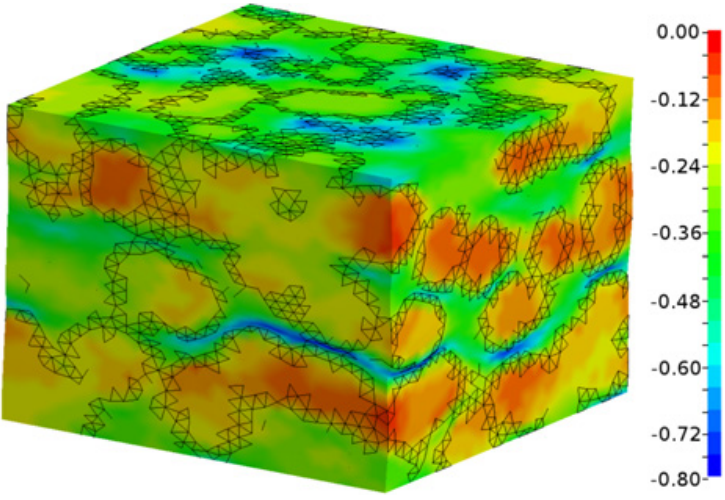

Fig. 2. Dynamic compression. Numerical $\varepsilon_{y}$ contours obtained for a macroscopic strain of $28 \%$ with the sample \#3.

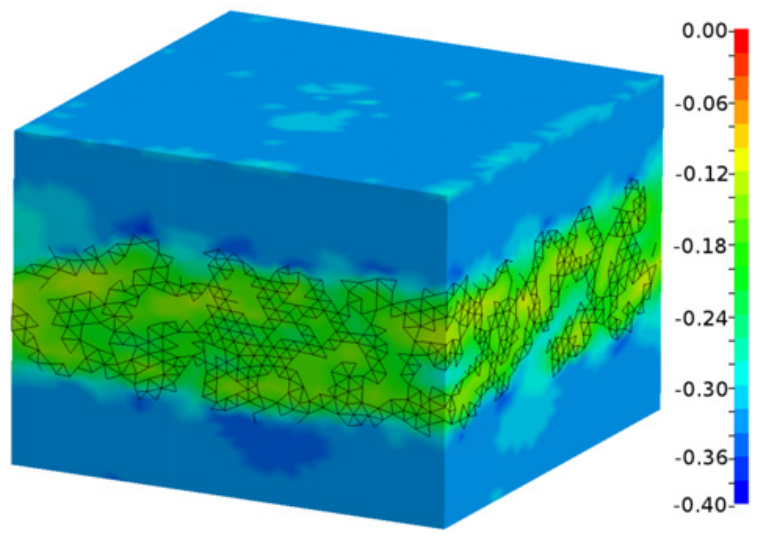

Fig. 3. Dynamic compression. Numerical $\varepsilon_{y}$ contours obtained for a macroscopic strain of $28 \%$ with the sample \#5.

impactor whereas the bottom of the sample has been fixed from axial displacement. The cube was discretized by more than 160000 linear tetrahedron elements which overcome numerical problem such as negative volumes [10].

\subsection{Influence of the heterogeneities on the mesoscopic behavior}

The interest of studying virtual volumes of foam is firstly to determine the influence of the organized system of heterogenities on the mesoscopic behavior and to observe strain localizations. At the mesoscopic scale, the numerical results were analyzed by using the contours of the axial strain along the vertical axis. The influence of the number, the position and the size of the heterogeneities has been studied and only representative results are presented for mescoscopic observations. The other results confirmed trends which are explained hereinafter.

With the sample \#3, i.e. with large heterogeneities, the localization of the strain can be clearly observed in the Figure 2. For a macroscopic strain of $28 \%$ and for a strain rate value of $130 \mathrm{~s}^{-1}$, the axial strain is localized in the less dense finite elements between heterogeneities and can reach a maximum of $79 \%$. The thinnest the space between two heterogeneities is, the largest the axial strain is.

With the sample \#5, i.e. with heterogeneities in the middle of the sample, the strain localization can be observed at the bottom and the top of the sample (Figure 3). 


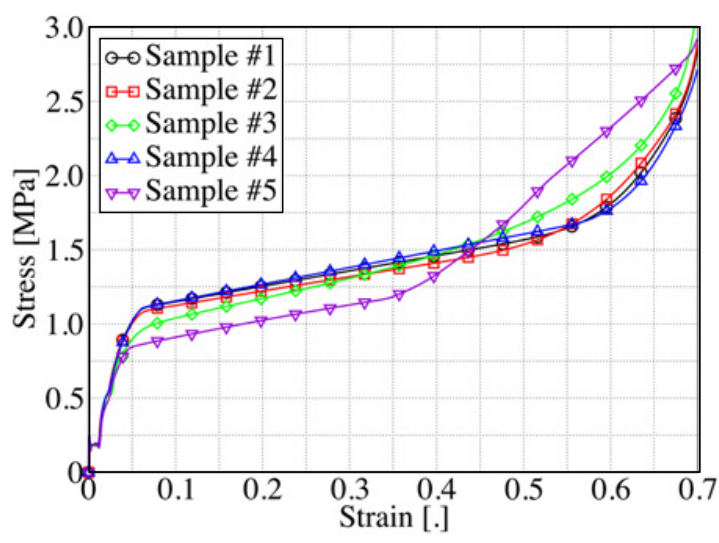

Fig. 4. Numerical results. Macroscopic behavior.

Three zones can be observed, the middle one for which the axial strain is about $18 \%$ and the bottom and the top ones with an axial strain of $32 \%$. The maximum of the axial strain is less important than previously and reaches a maximum of $40 \%$.

Finally, this shows that even if a difference of $50 \mathrm{~kg} \cdot \mathrm{m}^{-3}$ is applied between the heterogeneities and the rest of the volume for each virtual volume, the strain is localized in the less dense spaces between large heterogeneities.

\subsection{Influence of the heterogeneities on the macroscopic behavior}

The influence of the organized system of heterogeneities has been then studied on the macroscopic behavior.

Stress-strain curves are plotted for the studied virtual volumes in Figure 4. With foam samples constituted by a difference of $50 \mathrm{~kg} \cdot \mathrm{m}^{-3}$ between the heterogeneities and the rest of the volume, the macroscopic behavior is nearly the same whatever the number and the size of the heterogeneities in the foam sample.

It also can be seen in figure 4 that the macroscopic behavior is only different when the volume ratio of heterogeneities in the middle of the volume is sufficiently important, i.e. sample \#5. The heterogeneous zones are no more at the mesoscopic scale but have been fused to the macroscopic scale and the virtual sample with mesoscopic heterogeneities can be now considered as a multi-layered volume of foam.

In consequence, the only way to modify the macroscopic behavior of foam in the loading case of uni-axial compressive tests is to concentrate density heterogeneities and to pile up layers of different densities.

\section{Dynamic compression of multi-layered foams}

The previous numerical study showed the potential of multi-layered foams to modify the macroscopic behavior of foam in the case of dynamic compressive tests. Dynamic compressive tests have been performed with an original Flywheel device [8] which allows a quasi-constant strain
Table 2. Characteristics of the experimental samples of foam for compression tests.

\begin{tabular}{ccccc}
\hline & $\begin{array}{c}\text { Densities } \\
\text { layer \#1 } \\
{\left[\mathrm{kg} \cdot \mathrm{m}^{-3}\right]}\end{array}$ & $\begin{array}{c}\text { layer \#2 } \\
{\left[\mathrm{kg} \cdot \mathrm{m}^{-3}\right]}\end{array}$ & $\begin{array}{c}\text { layer \#3 } \\
{\left[\mathrm{kg} \cdot \mathrm{m}^{-3}\right]}\end{array}$ & $\begin{array}{c}\text { mean } \\
{\left[\mathrm{kg} \cdot \mathrm{m}^{-3}\right]}\end{array}$ \\
\hline Homogeneous & - & - & - & $94 \pm 2$ \\
$60-90-110$ & $64 \pm 3$ & $86 \pm 6$ & $112 \pm 5$ & $89 \pm 1$ \\
$110-40-110$ & $109 \pm 3$ & $42 \pm 3$ & $112 \pm 5$ & $89 \pm 1$ \\
$60-130-60$ & $62 \pm 2$ & $130 \pm 4$ & $67 \pm 2$ & $87 \pm 2$ \\
\hline
\end{tabular}

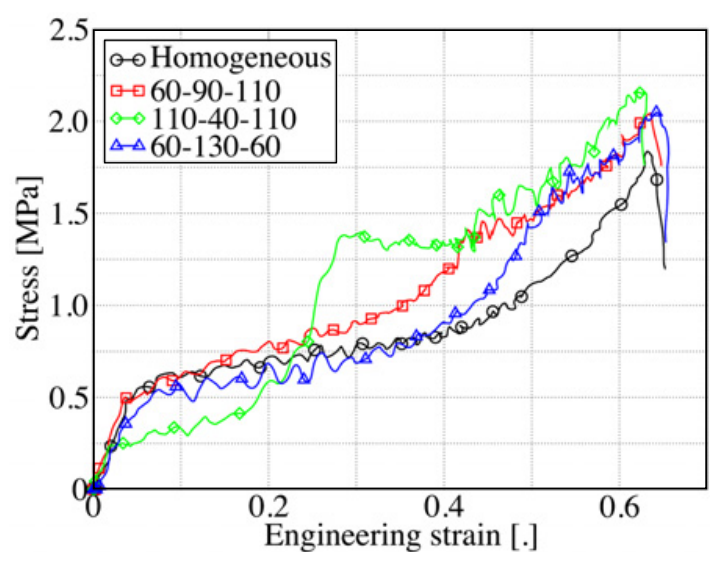

Fig. 5. Experimental results. Stress-strain curves.

rate during the test. The macroscopic strain rate chosen for this study is fixed to $110 \mathrm{~s}^{-1}$. Only the results at the macroscopic scale will be discussed afterwards because the mesoscopic results confirmed the results obtained previously.

One homogeneous and three different multi-layered foam samples with a size of approximately $30 \times 30 \times$ $30 \mathrm{~mm}^{3}$ were manufactured for this study and their characteristics are presented in the table 2. The thickness of the three layers is equal to one third of the sample thickness. The experiments have been performed on five samples of each kind of foam in order to check the repeatability. In order to compare the results of each sample, the mean density of each one has been adjusted to approximately $100 \mathrm{~kg} \cdot \mathrm{m}^{-3}$.

\subsection{Experimental results}

The stress-strain curves of each sample are plotted in Figure 5. It can be seen that the macroscopic behavior of the multi-layered samples is different from each other and from the homogeneous one. The steps of the stress plateau can be easily observed corresponding to the value of density which constitutes the layer of the sample. For instance, the stress plateau of the $40 \mathrm{~kg} \cdot \mathrm{m}^{-3}$ layer can be observed in the green curve (circle marks) at the beginning of the compressive test, i.e. when the strain is lower than 0.2 . Following the steps of stress plateau and densification of the $40 \mathrm{~kg} \cdot \mathrm{m}^{-3}$ layer, i.e. when the strain is in [0.3:0.6], the stress plateau of both $110 \mathrm{~kg} \cdot \mathrm{m}^{-3}$ layers can be 


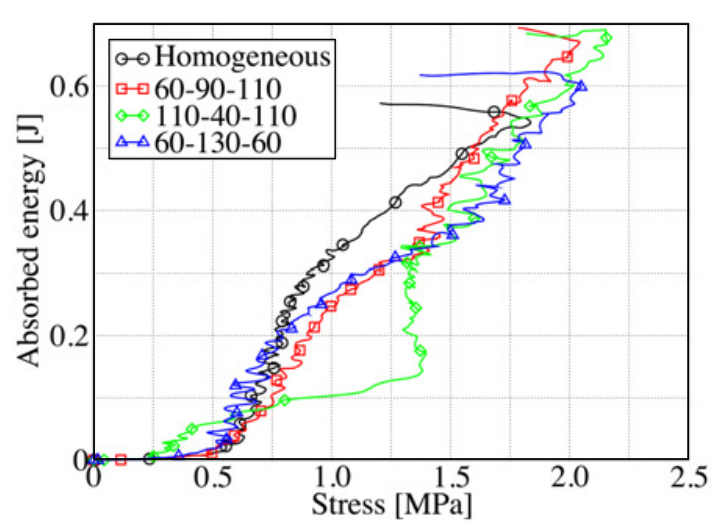

Fig. 6. Experimental results. Absorbed energy-stress curves.

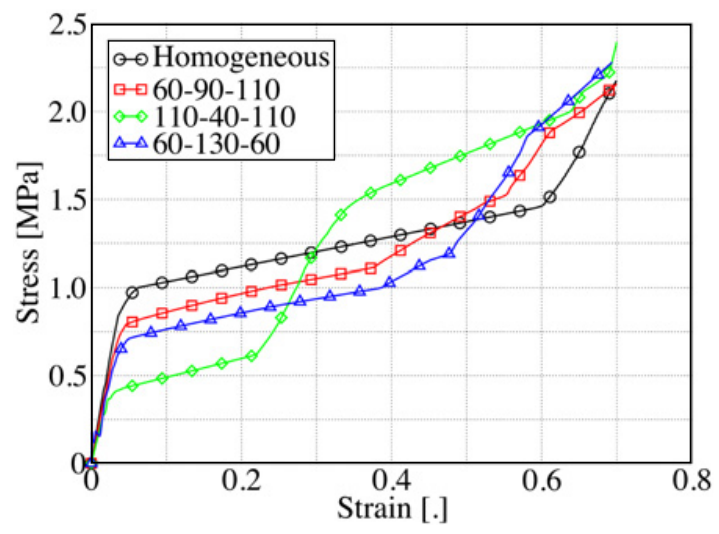

Fig. 7. Numerical results. Stress-strain curves.

observed. One can also note that the length of the stress plateau is directly related to the thickness of its layer. Moreover, the order of the constituted layers does not have any influence on the macroscopic behavior in this case of compressive tests.

These different macroscopic behaviors involve different capacity to absorb energy. The absorbed energy versus stress is plotted for each sample in Figure 6 and show the interest of multi-layered foam to gradually absorb energy. When an homogeneous foam is tested, the foam does not absorb the energy before a stress of $0.5 \mathrm{MPa}$ whereas energy is absorbed from $0.2 \mathrm{MPa}$ with the '11040-110' sample. However, this latter foam is less efficient than others when the $40 \mathrm{~kg} \cdot \mathrm{m}^{-3}$ layer reaches the step of densification, step during which a small quantity of energy is absorbed. This is due to the too large difference in density between the layers. Indeed, the '60-90-110' sample (red curve, square marks) for which the density is gradually increased seems to be the most adapted to impacts for which a gradual energy absorption is required.

\subsection{Numerical results}

FE simulation of the dynamic compressive tests were performed using LS-Dyna software with the same input data as in section 2. The numerical results are presented in terms of the stress-strain and the absorbed energy versus stress curves in the Figures 7 and 8. The macroscopic behavior of each multi-layered sample is well described

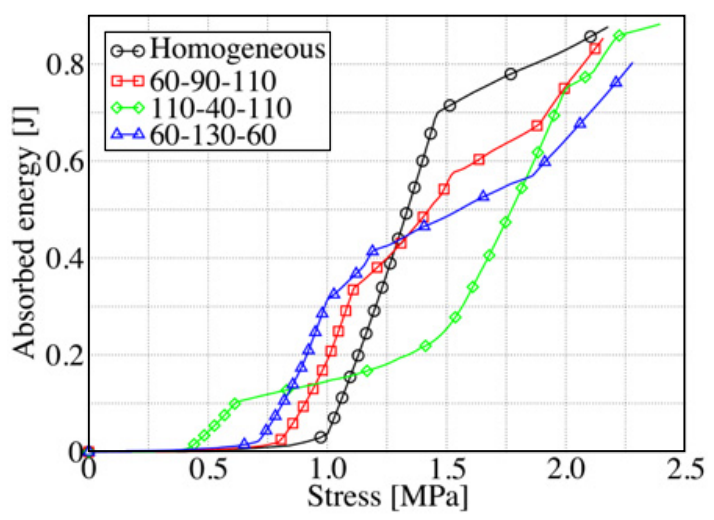

Fig. 8. Numerical results. Absorbed energy-stress curves.

by the FE simulation (figure 7) and one can easily see the different stress levels in each layer. Even if the trends are the same with the experimental dynamic compressive tests, the stress level of the first step of the test, i.e. when the strain is approximately lower than 0.4 , is overestimated by the FE simulation with all the samples. This difference comes from a numerical artefact which is due to the use of numerical damping in the LS-Dyna software to overcome negative volumes in the finite elements $[10,11]$. A numerical stiffness which is added to soft finite elements involves an increase of the stress level in the elastic and plateau stages. With this kind of multi-layered foams, the use of this numerical stiffness is necessary to prevent the soft layers from completely collapsing before the deformation of other layers.

Finally, these compressive tests exhibit the capabilities of this kind of structured foam to dissipate gradually the impact energy.

\section{Dynamic indentation of multi-layered foams}

The use of multilayered foams in dynamic compression has shown that the deformation is first localized in the softest layers whatever the position of the layers. The capabilities of these foams subjected to dynamic indentation have then been studied to highlight the advantages of such structures. Dynamic indentation tests have been performed with a drop tower with a $1.92 \mathrm{~kg}$ hemisphericalshape projectile. The drop height was fixed to $0.8 \mathrm{~m}$ and the impact velocity was about $4.0 \mathrm{~m} \cdot \mathrm{s}^{-1}$ which involves an impact energy of $15.36 \mathrm{~J}$. One homogeneous and three different multi-layered foam samples with a size of approximately $90 \times 30 \times 30 \mathrm{~mm}^{3}$ were manufactured for this study and their characteristics are presented in the table 3. In order to compare the results between each other, the normalized indentation has been defined as the indentation depth over the heigth of the sample. High speed cameras have also been used to capture images during the impact.

The experimental results has been first analyzed thanks to the Digital Image Correlation (DIC) technique in order to observe mesoscopic behavior in the samples. The software CORRELI Q4 has been used for the study [12]. A 
Table 3. Characteristics of the experimental samples of foam for indentation tests.

\begin{tabular}{|c|c|c|c|c|}
\hline Sample & $\begin{array}{c}\text { Densities } \\
\text { layer \#1 } \\
{\left[\mathrm{kg} \cdot \mathrm{m}^{-3}\right]}\end{array}$ & $\begin{array}{c}\text { layer \#2 } \\
{\left[\mathrm{kg} \cdot \mathrm{m}^{-3}\right]}\end{array}$ & $\begin{array}{c}\text { layer \#3 } \\
{\left[\mathrm{kg} \cdot \mathrm{m}^{-3}\right]}\end{array}$ & $\begin{array}{c}\text { mean } \\
{\left[\mathrm{kg} \cdot \mathrm{m}^{-3}\right]}\end{array}$ \\
\hline Homogeneous & - & - & - & $97 \pm 1$ \\
\hline $60-90-110$ & $70 \pm 2$ & $91 \pm 2$ & $120 \pm 2$ & $102 \pm 2$ \\
\hline $110-40-110$ & $116 \pm 3$ & $46 \pm 3$ & $123 \pm 3$ & $101 \pm 2$ \\
\hline $60-130-60$ & $65 \pm 2$ & $130 \pm 3$ & $73 \pm 2$ & $99 \pm 2$ \\
\hline
\end{tabular}

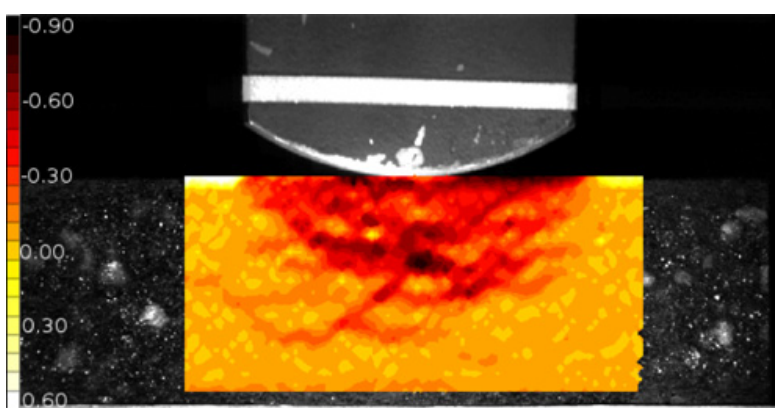

Fig. 9. Dynamic indentation of an homogeneous sample for a normalized indentation of $28 \% . \varepsilon_{y}$ contours obtained with the DIC technique.

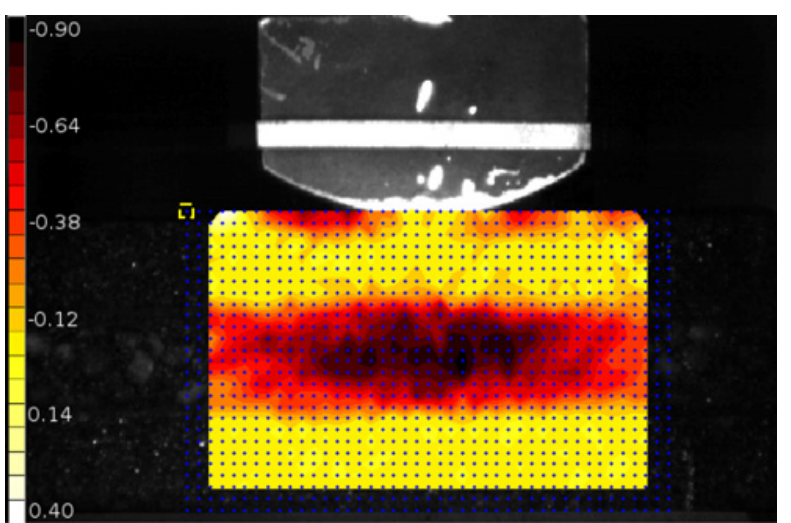

Fig. 10. Dynamic indentation of a 110-40-110 sample for a normalized indentation of $28 \% . \varepsilon_{y}$ contours obtained with the DIC technique.

Zone Of Interest (ZOI) of 8 pixels has been used for this study.

In Figure 9, the strain localization can be observed just below the projectile for the homogeneous sample. The darkest zones in this image match with the less dense polypropylene beads and the strain can reach $90 \%$. This show the density heterogeneity of the beads in such material [13]. Tension phenomenom can also be observed at the top of the sample and on either side of the projectile and fracture of the foam appears for a normalized indentation of $42 \pm 4 \%$.

In the figure 10, the strain localization can be observed in lowest density layer, i.e. the middle one and reaches a value of $90 \%$ whereas the strain level is less than $12 \%$ in the top and the bottom layers. The strain heterogeneities observed in the middle layer match with the softest beads of the foam.
Table 4. Macroscopic comparison between foam samples for indentation tests.

\begin{tabular}{cccc}
\hline & $\begin{array}{c}\text { Initial } \\
\text { velocity } \\
{\left[\mathrm{m} \cdot \mathrm{s}^{-1}\right]}\end{array}$ & $\begin{array}{c}\text { Rebound } \\
\text { velocity } \\
{\left[\mathrm{m} \cdot \mathrm{s}^{-1}\right]}\end{array}$ & $\begin{array}{c}\text { Absorbed } \\
\text { energy } \\
{[\%]}\end{array}$ \\
\hline Homogeneous & $\approx 4.0$ & $1.76 \pm 0.01$ & 80.6 \\
$60-90-110$ & $\approx 4.0$ & $1.87 \pm 0.04$ & 78.1 \\
$110-40-110$ & $\approx 4.0$ & $2.07 \pm 0.09$ & 73.2 \\
$60-130-60$ & $\approx 4.0$ & $2.02 \pm 0.04$ & 74.5 \\
\hline
\end{tabular}

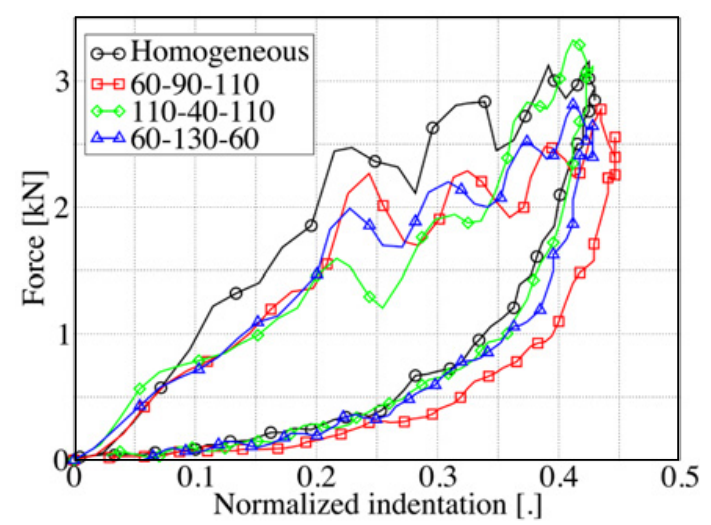

Fig. 11. Force comparison between foam samples in the case of a dynamic indentation.

The damping efficency of each sample of foams has therefore been compared by using the incident and the rebound velocities of the projectile. The results are presented in Table 4. Even if the density of each sample is about $100 \mathrm{~kg} \cdot \mathrm{m}^{-3}$, there are some macroscopic differences to damp the projectile impact. The best damping capacities are obtained with the homogeneous sample which can absorb more than $80 \%$ of the impact energy whereas the absorbed energy over the impact energy is about $73 \%$ with the 110-40-110 foam sample.

However, the absorbed energy can constitute an unique criterion to select foams for passive security. Indeed, the variation of the force and of its work can be supplementary criteria.

In the figure 11 , the force is plotted versus the normalized indentation. On each representative curve of a kind of sample, the signal is perturbed by oscillations are due to wave reflection inside the projectile. However, these oscillations do not prevent the analysis and the comparison of the curves. First, the homogeneous sample shows an increase in the force level for a given normalized indentation. Indeed the difference between the homogeneous one and the others is about $25 \%$ for a normalized indentation of 0.2. For a range of the normalized force of [0.2:0.3], the lowest level of force is observed in the least dense of the 110-40-110 sample. However, the force level of this sample reaches more than $3 \mathrm{kN}$ for the deepest indentation, higher than other samples. This is due to the heaviest layers of the foam, i.e. $110 \mathrm{~kg} \cdot \mathrm{m}^{-3}$, for which a higher stress plateau is noticed in Figure 5. A better compromise to 
limit the force during the entire impact duration is to use the 60-90-110 and the 60-130-60 samples for which the maximum force is about $2.5 \mathrm{MPa}$. For the latter one, even if the heavier layer is $130 \mathrm{~kg} \cdot \mathrm{m}^{-3}$, the force level does not exceed this value thanks to the maximum normalized indentation which is lower than 0.45 . In the case of deeper indentation, one should therefore be careful of the increase of the force level.

Finally, this experimental study shows the interest of such multi-layered foams to modify and limit the force level during the impact.

\section{Conclusion}

The polypropylene foam used for this study shows a multiscale heterogeneity. The mesoscopic heterogeneity, i.e. at the beads scale, has been studied numerically by creating virtual volumes of foam. The influence of the heterogeneities on the mesoscopic and the macroscopic behaviors has been observed and discussed. Thanks to this numerical work, strain localizations can easily be obtained and are mainly due to the thinnest spaces between heterogeneities. According to the macroscopic behavior, the only way to modify the dynamic behavior is to use multi-layered samples of foam with different densities for each layer.

A second part of the study dealt with the dynamic behavior of these kinds of foam subjected to compressive tests. Experiments show the interest of these structured foams to improve their damping capacities by dissipating the energy gradually. The strain is localized from the softest to the most rigid layers of foam and the macroscopic behavior matches the behavior of the softest to the most rigid layers sequentially.

Finally, the structured foams have been tested in the case of dynamic indentation. The strain localization is observed below the impactor and firstly in the softest layers. The conclusive points of this indentation tests matches with the ones of the compressive tests, i.e. the macroscopic behavior can be interpreted as a succesion of layer's behaviors.

\section{References}

1. NJ Mills, C Fitzgerald, A Gilchrist, R Verdejo. Compos Sci Technol. 63 (2003) 2389-2400.

2. LJ Gibson, MF Ashby. Cellular solids. Structure and properties. (Cambridge University Press, 1997) 532 pages.

3. MA Forero Rueda, L Cui, MD Gilchrist. Mater Des. 30 (2009) 3405-3413.

4. L Cui, S Kiernan, MD Gilchrist. Mater Sci Eng A. 507 (2009) 215-225.

5. EA Flores-Johnson, GM Li. Int J Sol Struct. 47 (2010) 1987-1995.

6. UE Osturk, G Anlas. Mater Des. 32 (2011) 773-780.

7. P Viot, F Beani, JL Lataillade. J Mater Sci. 40 (2005) 5829-5837.

8. R Bouix, P Viot, JL Lataillade. Int J Imp Eng 36 (2009) 329-342.

9. P Viot. Int J Imp Eng 36 (2009) 975-989.

10. O Hallquist. LS-Dyna Keyword user's manual. (Livermore Software Technology Corporation, 2007) 2206 pages.

11. A Hirth, P DuBois, $\mathrm{K}$ Weimar. Improvement of LSDyna Material Law 83 (Fu Chang) for the Industrial Simulation of Reversible Energy-Absorbing Foams. CAD-FEM Users Meeting (1998).

12. S Roux, F Hild, P Viot, D Bernard. Compos A: Appl Sci Manuf 39 (2008) 1253-1265.

13. P Viot, E Plougonven, D Bernard. Compos A: Appl Sci Manuf 39 (2008) 1266-1281. 